\title{
A NEW CHARACTERIZATION OF TRIEBEL-LIZORKIN SPACES ON $\mathbb{R}^{n}$
}

\author{
DAChUn Yang, Wen Yuan, AND YuAN ZhOU*
}

Abstract: In this paper, the authors characterize the Triebel-Lizorkin space $\dot{F}_{p, q}^{\alpha}\left(\mathbb{R}^{n}\right)$ via a new square function

$$
S_{\alpha, q}(f)(x)=\left\{\sum_{k \in \mathbb{Z}} 2^{k \alpha q}\left|\frac{1}{\left|B\left(x, 2^{-k}\right)\right|} \int_{B\left(x, 2^{-k}\right)}[f(x)-f(y)] d y\right|^{q}\right\}^{1 / q},
$$

where $f \in L_{\text {loc }}^{1}\left(\mathbb{R}^{n}\right) \cap \mathcal{S}^{\prime}\left(\mathbb{R}^{n}\right), x \in \mathbb{R}^{n}, \alpha \in(0,2)$ and $p, q \in(1, \infty]$. Similar characterizations are also established for Triebel-Lizorkin spaces $\dot{F}_{p, q}^{\alpha}\left(\mathbb{R}^{n}\right)$ with $\alpha \in(0, \infty) \backslash 2 \mathbb{N}$ and $p, q \in(1, \infty]$, and for Besov spaces $\dot{B}_{p, q}^{\alpha}\left(\mathbb{R}^{n}\right)$ with $\alpha \in(0, \infty) \backslash 2 \mathbb{N}, p \in(1, \infty]$ and $q \in(0, \infty]$.

2010 Mathematics Subject Classification: Primary: 46E35; Secondary: 42B25, $42 \mathrm{~B} 35$.

Key words: Triebel-Lizorkin space, Besov space, square function, Calderón reproducing formula.

\section{Introduction}

It is well known that the fractional Sobolev space $\dot{W}^{\alpha, p}\left(\mathbb{R}^{n}\right)$ with $\alpha \in(0,1)$ and $p \in(1, \infty)$ can be characterized by the square function $s_{\alpha}$, defined by setting, for all $x \in \mathbb{R}^{n}$ and $f \in L_{\text {loc }}^{1}\left(\mathbb{R}^{n}\right) \cap \mathcal{S}^{\prime}\left(\mathbb{R}^{n}\right)$,

$$
s_{\alpha}(f)(x):=\left\{\int_{0}^{\infty}\left[f_{B(x, t)}|f(x)-f(y)| d y\right]^{2} \frac{d t}{t^{1+2 \alpha}}\right\}^{1 / 2},
$$

Dachun Yang is supported by the National Natural Science Foundation (Grant No. 11171027) of China and Program for Changjiang Scholars and Innovative Research Team in University of China. Wen Yuan is supported by the National Natural Science Foundation (Grant No. 11101038) of China. Yuan Zhou is supported by Program for New Century Excellent Talents in University of China, National Natural Science Foundation of China (Grant No. 11201015) and the Academy of Finland grant 120972.

* Corresponding author. 
where above and in what follows, for any $g \in L_{\text {loc }}^{1}\left(\mathbb{R}^{n}\right)$ and ball $B \subset \mathbb{R}^{n}$,

$$
f_{B} g(y) d y:=\frac{1}{|B|} \int_{B} g(y) d y
$$

and $B(x, t)$ denotes the ball of $\mathbb{R}^{n}$ with the center $x \in \mathbb{R}^{n}$ and $t \in$ $(0, \infty)$; see, for example, [16], [12], [13], [17]. However, when $\alpha \geq 1$ and $p \in(1, \infty)$, the above square function fails to characterize $\dot{W}^{\alpha, p}\left(\mathbb{R}^{n}\right)$; indeed, if $f \in L_{\text {loc }}^{1}\left(\mathbb{R}^{n}\right)$ and $\left\|s_{\alpha}(f)\right\|_{L^{p}\left(\mathbb{R}^{n}\right)}<\infty$, then $f$ must be a constant function (see, for example, [6, Section 4]).

Recently, Alabern, Mateu and Verdera $[\mathbf{1}]$ characterized the fractional Sobolev space $\dot{W}^{\alpha, p}\left(\mathbb{R}^{n}\right)$ for $\alpha \in(0,2)$ and $p \in(1, \infty)$ via a new square function defined by setting, for all $f \in L_{\text {loc }}^{1}\left(\mathbb{R}^{n}\right) \cap \mathcal{S}^{\prime}\left(\mathbb{R}^{n}\right)$ and $x \in \mathbb{R}^{n}$,

$$
S_{\alpha}(f)(x):=\left\{\int_{0}^{\infty}\left|f_{B(x, t)}[f(x)-f(y)] d y\right|^{2} \frac{d t}{t^{1+2 \alpha}}\right\}^{1 / 2} .
$$

In particular, $S_{1}$-function characterizes the Sobolev space $\dot{W}^{1, p}\left(\mathbb{R}^{n}\right)$. Comparing $S_{\alpha}$ with $s_{\alpha}$, we see that the only difference is that $\mid f(x)-$ $f(y) \mid$ appearing in the definition of $s_{\alpha}(f)$ is replaced by $f(x)-f(y)$ in that of $S_{\alpha}(f)$. Such a slight difference leads to a quite different conclusion in the characterization of (fractional) Sobolev spaces. The main point, as first observed by Wheeden in [15] (see also [16]), when studying the Lipschitz-type (Besov) spaces, and later independently by Alabern, Mateu and Verdera in [1] , is that $S_{\alpha}$-function provides smoothness up to order 2 in the following sense: for all $f \in C^{2}\left(\mathbb{R}^{n}\right)$ and $t \in(0,1)$,

$$
f_{B(x, t)}[f(x)-f(y)] d y=O\left(t^{2}\right), \quad x \in \mathbb{R}^{n},
$$

which follows from the Taylor expansion of order 2

$$
f(y)=f(x)+\nabla f(x) \cdot(x-y)+O\left(|x-y|^{2}\right), \quad x, y \in \mathbb{R}^{n} .
$$

The purpose of this paper is to show that the above observation further leads to a new characterization of Triebel-Lizorkin spaces with reasonable parameters. We denote by $\dot{F}_{p, q}^{\alpha}\left(\mathbb{R}^{n}\right)$ the classical homogeneous Triebel-Lizorkin space while $F_{p, q}^{\alpha}\left(\mathbb{R}^{n}\right)$ the inhomogeneous TriebelLizorkin space for all reasonable parameters; see Section 2 for their definitions. Moreover, we introduce the following function spaces of TriebelLizorkin type via a variant of the above square function $S_{\alpha}$. 
Definition 1.1. Let $\alpha \in(0,2)$ and $q \in(0, \infty]$.

(i) If $p \in(0, \infty)$, the space $S \dot{F}_{p, q}^{\alpha}\left(\mathbb{R}^{n}\right)$ is defined as the collection of all functions $f \in L_{\text {loc }}^{1}\left(\mathbb{R}^{n}\right) \cap \mathcal{S}^{\prime}\left(\mathbb{R}^{n}\right)$ such that $\|f\|_{S \dot{F}_{p, q}^{\alpha}\left(\mathbb{R}^{n}\right)}:=\left\|S_{\alpha, q}(f)\right\|_{L^{p}\left(\mathbb{R}^{n}\right)}<$ $\infty$, where, for all $x \in \mathbb{R}^{n}$,

$$
S_{\alpha, q}(f)(x):=\left\{\sum_{k \in \mathbb{Z}} 2^{k \alpha q}\left|f_{B\left(x, 2^{-k}\right)}[f(x)-f(y)] d y\right|^{q}\right\}^{1 / q}
$$

with the usual modification made when $q=\infty$.

(ii) The space $S \dot{F}_{\infty, q}^{\alpha}\left(\mathbb{R}^{n}\right)$ is defined as the collection of all functions $f \in L_{\mathrm{loc}}^{1}\left(\mathbb{R}^{n}\right) \cap \mathcal{S}^{\prime}\left(\mathbb{R}^{n}\right)$ such that

$$
\begin{aligned}
& \|f\|_{S \dot{F}_{\infty, q}^{\alpha}\left(\mathbb{R}^{n}\right)} \\
& :=\sup _{x \in \mathbb{R}^{n}} \sup _{\ell \in \mathbb{Z}}\left\{f_{B\left(x, 2^{-\ell}\right)} \sum_{k \geq \ell} 2^{k \alpha q}\left|f_{B\left(y, 2^{-k}\right)}[f(y)-f(z)] d z\right|^{q} d y\right\}^{1 / q}<\infty
\end{aligned}
$$

with the usual modification made when $q=\infty$.

(iii) If $p \in(1, \infty]$, the inhomogeneous space $S F_{p, q}^{\alpha}\left(\mathbb{R}^{n}\right)$ is defined by

$$
S F_{p, q}^{\alpha}\left(\mathbb{R}^{n}\right):=L^{p}\left(\mathbb{R}^{n}\right) \cap S \dot{F}_{p, q}^{\alpha}\left(\mathbb{R}^{n}\right)
$$

with its norm $\|f\|_{S F_{p, q}^{\alpha}\left(\mathbb{R}^{n}\right)}:=\|f\|_{L^{p}\left(\mathbb{R}^{n}\right)}+\|f\|_{S \dot{F}_{p, q}^{\alpha}\left(\mathbb{R}^{n}\right)}$ for all $f \in$ $S F_{p, q}^{\alpha}\left(\mathbb{R}^{n}\right)$.

In the above definition, $\mathcal{S}\left(\mathbb{R}^{n}\right)$ denotes the space of all Schwartz functions and $\mathcal{S}^{\prime}\left(\mathbb{R}^{n}\right)$ its topological dual, namely, the space of all Schwartz distributions. Recall that $f \in L_{\text {loc }}^{1}\left(\mathbb{R}^{n}\right) \cap \mathcal{S}^{\prime}\left(\mathbb{R}^{n}\right)$ means that $f \in L_{\text {loc }}^{1}\left(\mathbb{R}^{n}\right)$ and the natural pair $\langle f, \varphi\rangle$ given by the integral $\int_{\mathbb{R}^{n}} f(x) \varphi(x) d x$ exists for all $\varphi \in \mathcal{S}\left(\mathbb{R}^{n}\right)$ and induces an element of $\mathcal{S}^{\prime}\left(\mathbb{R}^{n}\right)$.

Then the first main result of this paper reads as follows.

Theorem 1.1. Let $\alpha \in(0,2)$ and $p, q \in(1, \infty]$. Then $\dot{F}_{p, q}^{\alpha}\left(\mathbb{R}^{n}\right)=$ $S \dot{F}_{p, q}^{\alpha}\left(\mathbb{R}^{n}\right)$, with equivalent norms, and also $F_{p, q}^{\alpha}\left(\mathbb{R}^{n}\right)=S F_{p, q}^{\alpha}\left(\mathbb{R}^{n}\right)$, with equivalent norms.

Remark 1.1. Notice that to obtain Theorem 1.1, it is necessary to make the apriori assumption $f \in L_{\mathrm{loc}}^{1}\left(\mathbb{R}^{n}\right) \cap \mathcal{S}^{\prime}\left(\mathbb{R}^{n}\right)$ in Definition 1.1. Indeed, let $f\left(x_{1}, x_{2}\right):=e^{x_{1}} \sin x_{2}$ for $\left(x_{1}, x_{2}\right) \in \mathbb{R}^{2}$. Then $f$ is a harmonic function in the plane and hence by the mean value property,

$$
f_{B\left(x, 2^{-k}\right)}[f(x)-f(y)] d y=f(x)-f_{B\left(x, 2^{-k}\right)} f(y) d y=0
$$


for all $x \in \mathbb{R}^{2}$ and $k \in \mathbb{Z}$. So $f \in L_{\text {loc }}^{1}\left(\mathbb{R}^{2}\right)$ and $S_{\alpha, q}(f)=0 \in$ $L^{p}\left(\mathbb{R}^{n}\right)$ for all $\alpha, p, q$ as in Theorem 1.1. However, let $\varphi\left(x_{1}, x_{2}\right):=$ $e^{-x_{1} / 2} e^{-x_{2} / 2} \sin x_{2}$. Then $\varphi \in \mathcal{S}\left(\mathbb{R}^{2}\right)$ and $\int_{\mathbb{R}^{2}} f(x) \varphi(x) d x=\infty$, which implies that $f \notin \mathcal{S}^{\prime}\left(\mathbb{R}^{2}\right)$. Since $\dot{F}_{p, q}^{\alpha}\left(\mathbb{R}^{2}\right)$ is a subspace of $\mathcal{S}^{\prime}\left(\mathbb{R}^{2}\right)$ (or $\mathcal{S}^{\prime}\left(\mathbb{R}^{2}\right)$ modulo polynomials), we then conclude that $f \notin \dot{F}_{p, q}^{\alpha}\left(\mathbb{R}^{2}\right)$. In this sense, the assumption $f \in L_{\text {loc }}^{1}\left(\mathbb{R}^{n}\right) \cap \mathcal{S}^{\prime}\left(\mathbb{R}^{n}\right)$ in Definition 1.1 is necessary.

In what follows, the space $\dot{W}_{\text {loc }}^{2 N, 1}\left(\mathbb{R}^{n}\right)$ denotes the set of all functions that are locally in the homogeneous Sobolev space $\dot{W}^{2 N, 1}\left(\mathbb{R}^{n}\right)$. When $\alpha \in(2 N, 2 N+2)$ with $N \in \mathbb{N}:=\{1,2, \ldots\}$ and $q \in(0, \infty]$, as motivated by higher order Taylor expansions, for all $f \in \dot{W}_{\mathrm{loc}}^{2 N, 1}\left(\mathbb{R}^{n}\right) \cap \mathcal{S}^{\prime}\left(\mathbb{R}^{n}\right)$ and $x \in \mathbb{R}^{n}$, we set

$$
S_{\alpha, q}(f)(x):=\left\{\sum_{k \in \mathbb{Z}} 2^{k \alpha q}\left|f_{B\left(x, 2^{-k}\right)} R_{N}\left(y ; x, 2^{-k}\right) d y\right|^{q}\right\}^{1 / q},
$$

where, for all $x, y \in \mathbb{R}^{n}$,

$$
R_{N}\left(y ; x, 2^{-k}\right):=f(y)-f(x)-\sum_{j=1}^{N} \frac{1}{L_{j}} \Delta^{j} f(x)|y-x|^{2 j}
$$

with $L_{j}:=\Delta^{j}|x|^{2 j}$ for $j \in\{1, \ldots, N\}$; see [1] (also [15]) for more details. Similar to Definition 1.1, we introduce its following higher-order variant.

Definition 1.2. Let $\alpha \in(2 N, 2 N+2)$ with $N \in \mathbb{N}, q \in(0, \infty]$ and $S_{\alpha, q}(f)$ be as in (1.1).

(i) If $p \in(0, \infty)$, the space $S \dot{F}_{p, q}^{\alpha}\left(\mathbb{R}^{n}\right)$ is defined as the collection of all functions $f \in \dot{W}_{\text {loc }}^{2 N, 1}\left(\mathbb{R}^{n}\right) \cap \mathcal{S}^{\prime}\left(\mathbb{R}^{n}\right)$ such that $\|f\|_{S \dot{F}_{p, q}^{\alpha}\left(\mathbb{R}^{n}\right)}:=\left\|S_{\alpha, q}(f)\right\|_{L^{p}\left(\mathbb{R}^{n}\right)}<$ $\infty$ with the usual modification made when $q=\infty$.

(ii) The space $S \dot{F}_{\infty, q}^{\alpha}\left(\mathbb{R}^{n}\right)$ is defined as the collection of all functions $f \in \dot{W}_{\mathrm{loc}}^{2 N, 1}\left(\mathbb{R}^{n}\right) \cap \mathcal{S}^{\prime}\left(\mathbb{R}^{n}\right)$ such that

$$
\begin{aligned}
& \|f\|_{S \dot{F}_{\infty, q}^{\alpha}\left(\mathbb{R}^{n}\right)} \\
& \quad:=\sup _{x \in \mathbb{R}^{n}} \sup _{\ell \in \mathbb{Z}}\left\{f_{B\left(x, 2^{-\ell}\right)} \sum_{k \geq \ell} 2^{k \alpha q}\left|f_{B\left(y, 2^{-k}\right)} R_{N}\left(z ; y, 2^{-k}\right) d z\right|^{q} d y\right\}^{1 / q}<\infty
\end{aligned}
$$

with the usual modification made when $q=\infty$. 
(iii) If $p \in(1, \infty]$, the inhomogeneous space $S F_{p, q}^{\alpha}\left(\mathbb{R}^{n}\right)$ is defined by

$$
S F_{p, q}^{\alpha}\left(\mathbb{R}^{n}\right):=L^{p}\left(\mathbb{R}^{n}\right) \cap S \dot{F}_{p, q}^{\alpha}\left(\mathbb{R}^{n}\right)
$$

with its norm $\|f\|_{S F_{p, q}^{\alpha}\left(\mathbb{R}^{n}\right)}:=\|f\|_{L^{p}\left(\mathbb{R}^{n}\right)}+\|f\|_{S \dot{F}_{p, q}^{\alpha}\left(\mathbb{R}^{n}\right)}$ for all $f \in$ $S F_{p, q}^{\alpha}\left(\mathbb{R}^{n}\right)$.

Recall that, via the square function $S_{\alpha, 2}$, Alabern, Mateu and Verdera [1] also characterized the higher order Sobolev space $\dot{W}^{\alpha, p}$ for all $\alpha \in(2 N, 2 N+2)$ with $N \in \mathbb{N}$ and $p \in(1, \infty)$. We extend this as follows.

Theorem 1.2. Let $N \in \mathbb{N}, \alpha \in(2 N, 2 N+2)$ and $p, q \in(1, \infty]$. Then $\dot{F}_{p, q}^{\alpha}\left(\mathbb{R}^{n}\right)=S \dot{F}_{p, q}^{\alpha}\left(\mathbb{R}^{n}\right)$, with equivalent norms, and also $F_{p, q}^{\alpha}\left(\mathbb{R}^{n}\right)=$ $S F_{p, q}^{\alpha}\left(\mathbb{R}^{n}\right)$, with equivalent norms.

The paper is organized as follows. In Section 2, we recall several notions and notation. In Section 3, we prove Theorems 1.1 and 1.2. In Section 4, we extend the above results to Besov spaces and also give some further remarks on the case $\alpha \in 2 \mathbb{N}$ and on the higher order TriebelLizorkin spaces on metric measure spaces.

Finally, we point out that the proofs of Theorems 1.1 and 1.2 below are totally different from the method used in $[\mathbf{1}]$. The method in $[\mathbf{1}]$ strongly depends on the theory of Fourier transforms and vector-valued singular integrals, while our approach heavily depends on some Calderón reproducing formulae, one of which is from Peetre [11] (see also Frazier and Jawerth [3] and Frazier, Jawerth and Weiss [5], or Lemma 3.1 below) and some others are constructed in this paper (see Lemma 3.2 below).

\section{Notation and definitions}

Let $\mathbb{Z}_{+}:=\{0\} \cup \mathbb{N}$. Denote by $\mathcal{S}\left(\mathbb{R}^{n}\right)$ the space of all Schwartz functions, whose topology is determined by a family of seminorms, $\left\{\|\cdot\|_{\mathcal{S}_{k, m}\left(\mathbb{R}^{n}\right)}\right\}_{k, m \in \mathbb{Z}_{+}}$, where, for all $k \in \mathbb{Z}_{+}, m \in(0, \infty)$ and $\varphi \in \mathcal{S}\left(\mathbb{R}^{n}\right)$,

$$
\|\varphi\|_{\mathcal{S}_{k, m}\left(\mathbb{R}^{n}\right)}:=\sup _{\alpha \in \mathbb{Z}_{+}^{n},|\alpha| \leq k} \sup _{x \in \mathbb{R}^{n}}(1+|x|)^{m}\left|\partial^{\alpha} \varphi(x)\right| .
$$

Here, for any $\alpha:=\left(\alpha_{1}, \ldots, \alpha_{n}\right) \in \mathbb{Z}_{+}^{n},|\alpha|:=\alpha_{1}+\cdots+\alpha_{n}$ and $\partial^{\alpha}:=$ $\left(\frac{\partial}{\partial x_{1}}\right)^{\alpha_{1}} \cdots\left(\frac{\partial}{\partial x_{n}}\right)^{\alpha_{n}}$. It is known that $\mathcal{S}\left(\mathbb{R}^{n}\right)$ forms a locally convex topological vector space. Denote by $\mathcal{S}^{\prime}\left(\mathbb{R}^{n}\right)$ the topological dual space of $\mathcal{S}\left(\mathbb{R}^{n}\right)$ endowed with the weak $*$-topology. In what follows, for every $\varphi \in \mathcal{S}\left(\mathbb{R}^{n}\right), t>0$ and $x \in \mathbb{R}^{n}$, set $\varphi_{t}(x):=t^{-n} \varphi\left(t^{-1} x\right)$.

For $p \in(0, \infty]$, denote by $L^{p}\left(\mathbb{R}^{n}\right)$ the Lebesgue space of order $p$. For $N \in \mathbb{N}$ and $p \in(1, \infty)$, denote by $\dot{W}^{N, p}\left(\mathbb{R}^{n}\right)$ the homogeneous Sobolev space of order $N$, namely, the collection of all measurable functions $f$ 
with their distributional derivatives $\partial^{\alpha} f \in L^{p}\left(\mathbb{R}^{n}\right)$, where $\alpha \in \mathbb{Z}_{+}^{n}$ and $|\alpha|=N$. Moreover, let

$$
\|f\|_{\dot{W}^{N, p}\left(\mathbb{R}^{n}\right)}:=\sum_{|\alpha|=N}\left\|\partial^{\alpha} f\right\|_{L^{p}\left(\mathbb{R}^{n}\right)} .
$$

Set $W^{N, p}\left(\mathbb{R}^{n}\right):=L^{p}\left(\mathbb{R}^{n}\right) \cap \dot{W}^{N, p}\left(\mathbb{R}^{n}\right)$ as the inhomogeneous Sobolev space with norm

$$
\|f\|_{W^{N, p}\left(\mathbb{R}^{n}\right)}:=\|f\|_{L^{p}\left(\mathbb{R}^{n}\right)}+\|f\|_{\dot{W}^{N, p}\left(\mathbb{R}^{n}\right)}
$$

for all $f \in W^{N, p}\left(\mathbb{R}^{n}\right)$. Denote by the space $L_{\text {loc }}^{1}\left(\mathbb{R}^{n}\right)$ the locally integrable function and similarly the space $\dot{W}_{\mathrm{loc}}^{N, 1}\left(\mathbb{R}^{n}\right)$.

Now we recall the notions of Triebel-Lizorkin and Besov spaces; see $[\mathbf{1 3}],[\mathbf{1 4}]$. In what follows, for any $\varphi \in L^{1}\left(\mathbb{R}^{n}\right), \widehat{\varphi}$ denotes the Fourier transform of $\varphi$, namely, for all $\xi \in \mathbb{R}^{n}$,

$$
\widehat{\varphi}(\xi):=\int_{\mathbb{R}^{n}} e^{-i \xi \cdot x} \varphi(x) d x .
$$

Definition 2.1. Let $\alpha \in(0, \infty), p, q \in(0, \infty]$ and $\varphi \in \mathcal{S}\left(\mathbb{R}^{n}\right)$ satisfy that

(2.1) $\operatorname{supp} \widehat{\varphi} \subset\left\{\xi \in \mathbb{R}^{n}: 1 / 2 \leq|\xi| \leq 2\right\}$ and

$$
|\widehat{\varphi}(\xi)| \geq \text { constant }>0 \text { if } 3 / 5 \leq|\xi| \leq 5 / 3 \text {. }
$$

(i) The homogeneous Triebel-Lizorkin space $\dot{F}_{p, q}^{\alpha}\left(\mathbb{R}^{n}\right)$ is defined as the collection of all $f \in \mathcal{S}^{\prime}\left(\mathbb{R}^{n}\right)$ such that $\|f\|_{\dot{F}_{p, q}^{\alpha}\left(\mathbb{R}^{n}\right)}<\infty$, where, when $p \in(0, \infty)$,

$$
\|f\|_{\dot{F}_{p, q}^{\alpha}\left(\mathbb{R}^{n}\right)}:=\left\|\left(\sum_{k \in \mathbb{Z}} 2^{k s q}\left|\varphi_{2^{-k}} * f\right|^{q}\right)^{1 / q}\right\|_{L^{p}\left(\mathbb{R}^{n}\right)},
$$

with the usual modification made when $q=\infty$, and

$$
\|f\|_{\dot{F}_{\infty, q}^{\alpha}\left(\mathbb{R}^{n}\right)}:=\sup _{x \in \mathbb{R}^{n}} \sup _{\ell \in \mathbb{Z}}\left\{f_{B\left(x, 2^{-\ell}\right)} \sum_{k \geq \ell} 2^{k \alpha q}\left|\varphi_{2^{-k}} * f(y)\right|^{q} d y\right\}^{1 / q},
$$

with the usual modification made when $q=\infty$.

When $p \in(1, \infty]$, the inhomogeneous Triebel-Lizorkin space $F_{p, q}^{\alpha}\left(\mathbb{R}^{n}\right)$ is defined by

$$
F_{p, q}^{\alpha}\left(\mathbb{R}^{n}\right):=L^{p}\left(\mathbb{R}^{n}\right) \cap \dot{F}_{p, q}^{\alpha}\left(\mathbb{R}^{n}\right)
$$

with the norm $\|f\|_{F_{p, q}^{\alpha}\left(\mathbb{R}^{n}\right)}:=\|f\|_{L^{p}\left(\mathbb{R}^{n}\right)}+\|f\|_{\dot{F}_{p, q}^{\alpha}\left(\mathbb{R}^{n}\right)}$ for all $f \in F_{p, q}^{\alpha}\left(\mathbb{R}^{n}\right)$. 
(ii) The homogeneous Besov space $\dot{B}_{p, q}^{\alpha}\left(\mathbb{R}^{n}\right)$ is defined as the collection of all $f \in \mathcal{S}^{\prime}\left(\mathbb{R}^{n}\right)$ such that $\|f\|_{\dot{B}_{p, q}^{\alpha}\left(\mathbb{R}^{n}\right)}<\infty$, where

$$
\|f\|_{\dot{B}_{p, q}^{\alpha}\left(\mathbb{R}^{n}\right)}:=\left(\sum_{k \in \mathbb{Z}} 2^{k s q}\left\|\varphi_{2^{-k}} * f\right\|_{L^{p}\left(\mathbb{R}^{n}\right)}^{q}\right)^{1 / q}
$$

with the usual modifications made when $p=\infty$ or $q=\infty$.

When $p \in(1, \infty]$, the inhomogeneous Besov space $B_{p, q}^{\alpha}\left(\mathbb{R}^{n}\right)$ is defined by

$$
B_{p, q}^{\alpha}\left(\mathbb{R}^{n}\right):=L^{p}\left(\mathbb{R}^{n}\right) \cap \dot{B}_{p, q}^{\alpha}\left(\mathbb{R}^{n}\right)
$$

with the $\operatorname{norm}\|f\|_{B_{p, q}^{\alpha}\left(\mathbb{R}^{n}\right)}:=\|f\|_{L^{p}\left(\mathbb{R}^{n}\right)}+\|f\|_{\dot{B}_{p, q}^{\alpha}\left(\mathbb{R}^{n}\right)}$ for all $f \in B_{p, q}^{\alpha}\left(\mathbb{R}^{n}\right)$.

Remark 2.1. Notice that if $\|f\|_{\dot{F}_{p, q}^{s}\left(\mathbb{R}^{n}\right)}=0$, then it is easy to see that $f$ is a polynomial. Denote by $\mathcal{P}\left(\mathbb{R}^{n}\right)$ the collection of all polynomials on $\mathbb{R}^{n}$. So the quotient space $\dot{F}_{p, q}^{s}\left(\mathbb{R}^{n}\right) / \mathcal{P}\left(\mathbb{R}^{n}\right)$ is a quasi-Banach space. By abuse of the notation, the space $\dot{F}_{p, q}^{s}\left(\mathbb{R}^{n}\right) / \mathcal{P}\left(\mathbb{R}^{n}\right)$ is always denoted by $\dot{F}_{p, q}^{s}\left(\mathbb{R}^{n}\right)$, and its element $[f]=f+\mathcal{P}\left(\mathbb{R}^{n}\right)$ with $f \in \dot{F}_{p, q}^{s}\left(\mathbb{R}^{n}\right)$ simply by $f$. Similar observation is also suitable to homogeneous Besov spaces.

Moreover, for $\alpha \in(0, \infty)$ and $p \in(1, \infty)$, the homogeneous fractional Sobolev space $\dot{W}^{\alpha, p}\left(\mathbb{R}^{n}\right)$ coincides with the Triebel-Lizorkin space $\dot{F}_{p, 2}^{\alpha}\left(\mathbb{R}^{n}\right)$ with equivalent norms; see [13] (or [14]) for this and the definition of $\dot{W}^{\alpha, p}\left(\mathbb{R}^{n}\right)$. Similarly, the inhomogeneous fractional Sobolev space $W^{\alpha, p}\left(\mathbb{R}^{n}\right)$ coincides with the Triebel-Lizorkin space $F_{p, 2}^{\alpha}\left(\mathbb{R}^{n}\right)$ with equivalent norms.

Throughout the whole paper, we denote by $C$ a positive constant which is independent of the main parameters, but it may vary from line to line. The symbol $A \lesssim B$ means that $A \leq C B$. If $A \lesssim B$ and $B \lesssim A$, we then write $A \sim B$. If $E$ is a subset of $\mathbb{R}^{n}$, we denote by $\chi_{E}$ the characteristic function of $E$. For any $a \in \mathbb{R},\lfloor a\rfloor$ denotes the maximal integer not larger than $a$.

\section{Proofs of the main results}

Theorems 1.1 and 1.2 follow from Remark 2.1 and the following Theorems 3.1 and 3.2 .

Theorem 3.1. Let $\alpha \in(0, \infty) \backslash 2 \mathbb{N}$ and $p, q \in(1, \infty]$. If $f \in \dot{F}_{p, q}^{\alpha}\left(\mathbb{R}^{n}\right)$, then there exists a polynomial $P_{f}$ such that $f+P_{f} \in S \dot{F}_{p, q}^{\alpha}\left(\mathbb{R}^{n}\right) ;$ moreover, $\left\|f+P_{f}\right\|_{S \dot{F}_{p, q}^{\alpha}\left(\mathbb{R}^{n}\right)} \leq C\|f\|_{\dot{F}_{p, q}^{\alpha}\left(\mathbb{R}^{n}\right)}$, where $C$ is a positive constant independent of $f$. 
To prove Theorem 3.1, we need the following Calderón reproducing formula established in [11, pp. 52-54] (see also [3, Remark 2.2]).

Lemma 3.1. For any $\varphi \in \mathcal{S}\left(\mathbb{R}^{n}\right)$ satisfying (2.1), there exists $\psi \in$ $\mathcal{S}\left(\mathbb{R}^{n}\right)$ satisfying (2.1) such that, for all $\xi \in \mathbb{R}^{n} \backslash\{0\}$,

$$
\sum_{j \in \mathbb{Z}} \widehat{\varphi}\left(2^{j} \xi\right) \widehat{\psi}\left(2^{j} \xi\right)=1 .
$$

Moreover, for every $f \in \mathcal{S}^{\prime}\left(\mathbb{R}^{n}\right)$, there exist polynomials $\left\{P_{j}\right\}_{j \in \mathbb{Z}}$ and $P_{f}$ such that

$$
f+P_{f}=\lim _{i \rightarrow-\infty}\left\{\sum_{j=i}^{\infty} \varphi_{2^{-j}} * \psi_{2^{-j}} * f+P_{i}\right\}
$$

in $\mathcal{S}^{\prime}\left(\mathbb{R}^{n}\right)$.

Proof of Theorem 3.1: We first assume that $\alpha \in(0,2)$ and $p, q \in(1, \infty]$. Notice that $\dot{F}_{p, q}^{\alpha}\left(\mathbb{R}^{n}\right) \subset L_{\text {loc }}^{1}\left(\mathbb{R}^{n}\right)$; see, for example, [10, Proposition 4.2] or [19, Proposition 5.1] for a proof. Let $f \in \dot{F}_{p, q}^{\alpha}\left(\mathbb{R}^{n}\right)$. Then $f \in$ $\mathcal{S}^{\prime}\left(\mathbb{R}^{n}\right) \cap L_{\text {loc }}^{1}\left(\mathbb{R}^{n}\right)$. Let $\varphi$ and $\psi$ be as in Lemma 3.1. Then (3.1) holds for $f$. Observe that $f \in \dot{F}_{p, q}^{\alpha}\left(\mathbb{R}^{n}\right)$ further implies that the degrees of the polynomials $\left\{P_{i}\right\}_{i \in \mathbb{Z}}$ in $(2.1)$ do not exceed $\lfloor\alpha-n / p\rfloor \leq 1$; see $[\mathbf{4}$, pp. 153-155] and [3]. Moreover, since $P_{i}$ has at most degree 1 for each $i$, we have

$$
P_{i}(x)-f_{B\left(x, 2^{-k}\right)} P_{i}(z) d z=0
$$

for all $x \in \mathbb{R}^{n}$ and $k \in \mathbb{Z}$. Moreover, as shown in [4, pp. 153-155], $f+P_{f}$ is the canonical representative of $f$ in the sense that if for $i \in$ $\{1,2\}, \varphi^{(i)}$ and $\psi^{(i)}$ satisfy $(2.1)$ and

$$
\sum_{k \in \mathbb{Z}} \widehat{\varphi^{(i)}}\left(2^{-k} \xi\right) \widehat{\psi^{(i)}}\left(2^{-k} \xi\right)=1
$$

for all $\xi \in \mathbb{R}^{n} \backslash\{0\}$, then $P_{f}^{(1)}-P_{f}^{(2)}$ is a polynomial of degree not more than $\lfloor\alpha-n / p\rfloor \leq 1$, where $P_{f}^{(i)}$ is as in (3.1) corresponding to $\left\{\varphi^{(i)}, \psi^{(i)}\right\}$ for $i \in\{1,2\}$. Also notice that for all $x \in \mathbb{R}^{n}$ and $k \in \mathbb{Z}$,

$$
P_{f}^{(1)}(x)-P_{f}^{(2)}(x)-f_{B\left(x, 2^{-k}\right)}\left[P_{f}^{(1)}(z)-P_{f}^{(2)}(z)\right] d z=0 .
$$

Let $\tilde{f}:=f+P_{f}$. Then by $(3.1)$, we have

$$
\widetilde{f}-\widetilde{f}_{B\left(\cdot, 2^{-k}\right)}=\sum_{j \in \mathbb{Z}}\left(\varphi_{2^{-j}}-\chi_{k} * \varphi_{2^{-j}}\right) * \psi_{2^{-j}} * f
$$


in $\mathcal{S}^{\prime}\left(\mathbb{R}^{n}\right)$. Here $\chi:=\frac{\chi_{B(0,1)}}{|B(0,1)|}$ and $\chi_{k}:=2^{k n} \chi\left(2^{k} \cdot\right)$. From the above discussion, it follows that $\widetilde{f}-\widetilde{f}_{B\left(\cdot, 2^{-k}\right)}$ is independent of the choices of $\varphi$ and $\psi$ satisfying $(2.1)$. Then it suffices to prove that, when $p \in(1, \infty)$ and $q \in(1, \infty]$,

$$
\begin{array}{r}
\left\{\int_{\mathbb{R}^{n}}\left[\sum_{k \in \mathbb{Z}} 2^{k \alpha q}\left(\sum_{j \in \mathbb{Z}}\left|\left(\varphi_{2^{-j}}-\chi_{k} * \varphi_{2^{-j}}\right) * \psi_{2^{-j}} * f(x)\right|\right)^{q}\right]^{\frac{p}{q}} d x\right\}^{\frac{1}{p}} \\
\lesssim\|f\|_{\dot{F}_{p, q}^{\alpha}\left(\mathbb{R}^{n}\right)}
\end{array}
$$

and that, when $p=\infty$ and $q \in(1, \infty]$, for all $x \in \mathbb{R}^{n}$ and $\ell \in \mathbb{Z}$,

$$
\begin{array}{r}
\left\{f_{B\left(x, 2^{-\ell}\right)} \sum_{k \geq \ell} 2^{k \alpha q}\left(\sum_{j \in \mathbb{Z}}\left|\left(\varphi_{2^{-j}}-\chi_{k} * \varphi_{2^{-j}}\right) * \psi_{2^{-j}} * f(y)\right|\right)^{q} d y\right\}^{\frac{1}{q}} \\
\lesssim\|f\|_{F_{\infty, q}^{\alpha}\left(\mathbb{R}^{n}\right)}
\end{array}
$$

Indeed, if (3.3) holds, then for each $k \in \mathbb{Z}$, we have

$$
\int_{\mathbb{R}^{n}}\left[\sum_{j \in \mathbb{Z}}\left|\left(\varphi_{2^{-j}}-\chi_{k} * \varphi_{2^{-j}}\right) * \psi_{2^{-j}} * f(x)\right|\right]^{p} d x<\infty,
$$

which further implies that (3.2) holds in $L^{p}\left(\mathbb{R}^{n}\right)$ and hence almost everywhere. Therefore, for every $k \in \mathbb{Z}$,

$$
\left|\widetilde{f}-\widetilde{f}_{B\left(\cdot, 2^{-k}\right)}\right| \leq \sum_{j \in \mathbb{Z}}\left|\left(\varphi_{2^{-j}}-\chi_{k} * \varphi_{2^{-j}}\right) * \psi_{2^{-j}} * f\right|
$$

almost everywhere, and hence $\|\widetilde{f}\|_{S \dot{F}_{p, q}^{\alpha}\left(\mathbb{R}^{n}\right)}$ is less than the left hand side of (3.3), which further implies that $\|\widetilde{f}\|_{S \dot{F}_{p, q}^{\alpha}\left(\mathbb{R}^{n}\right)} \lesssim\|f\|_{\dot{F}_{p, q}^{\alpha}\left(\mathbb{R}^{n}\right)}$. Similarly, if (3.4) holds, then (3.2) holds in $L_{\text {loc }}^{1}\left(\mathbb{R}^{n}\right)$ and hence almost everywhere and, moreover, an argument similar to above leads to $\|\widetilde{f}\|_{S \dot{F}_{\infty, q}^{\alpha}\left(\mathbb{R}^{n}\right)} \lesssim$ $\|f\|_{\dot{F}_{\infty, q}^{\alpha}\left(\mathbb{R}^{n}\right)}$.

To prove (3.3), we consider $\sum_{j \leq k}$ and $\sum_{j>k}$ separately. Notice that for any smooth function $\Phi$ on $\mathbb{R}$,

$$
\Phi(1)=\Phi(0)+\int_{0}^{1} \Phi^{\prime}(s) d s=\Phi(0)+\Phi^{\prime}(0)+\int_{0}^{1}(1-s) \Phi^{\prime \prime}(s) d s .
$$


Let $\Phi(s):=\varphi\left(2^{j} x+s z\right)$ for $s \in[0,1]$ and $x, z \in \mathbb{R}^{n}$. Then $\varphi\left(2^{j} x+z\right)=\varphi\left(2^{j} x\right)+(\nabla \varphi)\left(2^{j} x\right) z^{t}+\int_{0}^{1}(1-s) z\left(\nabla^{2} \varphi\right)\left(2^{j} x+s z\right) z^{t} d s$, where $z^{t}$ denotes the transpose of $z$. Therefore, when $j \leq k$, for all $x \in \mathbb{R}^{n}$,

$$
\begin{aligned}
\left|\chi_{k} * \varphi_{2^{-j}}(x)-\varphi_{2^{-j}}(x)\right| & =\left|f_{B(0,1)} 2^{j n}\left[\varphi\left(2^{j} x+2^{j-k} z\right)-\varphi\left(2^{j} x\right)\right] d z\right| \\
& =\left|f_{B\left(0,2^{j-k}\right)} 2^{j n}\left[\varphi\left(2^{j} x+z\right)-\varphi\left(2^{j} x\right)\right] d z\right| \\
& =\left|f_{B\left(0,2^{j-k}\right)} 2^{j n} \int_{0}^{1}(1-s) z\left(\nabla^{2} \varphi\right)\left(2^{j} x+s z\right) z^{t} d s d z\right| \\
& \lesssim 2^{2(j-k)} \frac{2^{j n}}{\left(1+\left|2^{j} x\right|\right)^{L}},
\end{aligned}
$$

where $L>n$. Hence

$$
\begin{aligned}
\left|\left(\chi_{k} * \varphi_{2^{-j}}-\varphi_{2^{-j}}\right) * \psi_{2^{-j}} * f(x)\right| & \lesssim 2^{2(j-k)} \int_{\mathbb{R}^{n}} \frac{2^{j n}}{\left(1+\left|2^{j} y\right|\right)^{L}}\left|\psi_{2^{-j}} * f(x-y)\right| d y \\
& \lesssim 2^{2(j-k)} M\left(\left|\psi_{2^{-j}} * f\right|\right)(x),
\end{aligned}
$$

where $M$ denotes the Hardy-Littlewood maximal function. Then, choosing $\delta \in(0,2-\alpha)$, by Hölder's inequality and $\alpha \in(0,2)$, we see that

$$
\begin{aligned}
\mathrm{I}_{1} & :=\left\{\int_{\mathbb{R}^{n}}\left[\sum_{k \in \mathbb{Z}} 2^{k \alpha q}\left(\sum_{j \leq k}\left|\left(\varphi_{2^{-j}}-\chi_{k} * \varphi_{2^{-j}}\right) * \psi_{2^{-j}} * f(x)\right|\right)^{q}\right]^{p / q} d x\right\}^{1 / p} \\
& \lesssim\left\{\int_{\mathbb{R}^{n}}\left[\sum_{k \in \mathbb{Z}} 2^{k \alpha q} \sum_{j \leq k} 2^{(2-\delta)(j-k) q}\left[M\left(\left|\psi_{2^{-j}} * f\right|\right)(x)\right]^{q}\right]^{p / q} d x\right\}^{1 / p} \\
& \lesssim\left\{\int_{\mathbb{R}^{n}}\left[\sum_{j \in \mathbb{Z}} 2^{j \alpha q}\left[M\left(\left|\psi_{2^{-j}} * f\right|\right)(x)\right]^{q}\right]^{p / q} d x\right\}^{1 / p},
\end{aligned}
$$


which, together with the Fefferman-Stein vector-valued maximal inequality (see [2]), further implies that

$$
\mathrm{I}_{1} \lesssim\left\{\int_{\mathbb{R}^{n}}\left[\sum_{j \in \mathbb{Z}} 2^{j \alpha q}\left|\psi_{2^{-j}} * f(x)\right|^{q}\right]^{p / q} d x\right\}^{1 / p} \lesssim\|f\|_{\dot{F}_{p, q}^{\alpha}\left(\mathbb{R}^{n}\right)} .
$$

Notice that when $j>k$, for all $x \in \mathbb{R}^{n}$, we always have

$$
\begin{aligned}
\left|\left(\chi_{k} * \varphi_{2^{-j}}-\varphi_{2^{-j}}\right) * \psi_{2^{-j}} * f(x)\right| & \leq\left|\chi_{k} * \varphi_{2^{-j}} * \psi_{2^{-j}} * f(x)\right|+\left|\varphi_{2^{-j}} * \psi_{2^{-j}} * f(x)\right| \\
& \leq \chi_{k^{*}} *\left[M\left(\left|\psi_{2^{-j}} * f\right|\right)\right](x)+M\left(\left|\psi_{2^{-j}} * f\right|\right)(x) \mid \\
& \lesssim M \circ M\left(\left|\psi_{2^{-j}} * f\right|\right)(x),
\end{aligned}
$$

where $M \circ M$ denotes the composition of $M$ and $M$. Then by $\alpha>0$, taking $\delta \in(0, \alpha)$ and applying Hölder's inequality, we obtain

$$
\begin{aligned}
\mathrm{I}_{2} & :=\left\{\int_{\mathbb{R}^{n}}\left[\sum_{k \in \mathbb{Z}} 2^{k \alpha q}\left(\sum_{j>k}\left|\left(\varphi_{2^{-j}}-\chi_{k} * \varphi_{2^{-j}}\right) * \psi_{2^{-j}} * f(x)\right|\right)^{q}\right]^{p / q} d x\right\}^{1 / p} \\
& \lesssim\left\{\int_{\mathbb{R}^{n}}\left[\sum_{k \in \mathbb{Z}} 2^{k(\alpha-\delta) q} \sum_{j>k} 2^{j \delta q}\left[M \circ M\left(\left|\psi_{2^{-j}} * f\right|\right)(x)\right]^{q}\right]^{p / q} d x\right\}^{1 / p} \\
& \lesssim\left\{\int_{\mathbb{R}^{n}}\left[\sum_{j \in \mathbb{Z}} 2^{j \alpha q}\left[M \circ M\left(\left|\psi_{2^{-j}} * f\right|\right)(x)\right]^{q}\right]^{p / q} d x\right\}^{1 / p},
\end{aligned}
$$

which, together with the Fefferman-Stein vector-valued maximal inequality, further implies that $\mathrm{I}_{2} \lesssim\|f\|_{\dot{F}_{p, q}^{\alpha}\left(\mathbb{R}^{n}\right)}$. This proves (3.3).

To prove (3.4), we consider $\sum_{j<\ell \leq k}, \sum_{\ell \leq j \leq k}$ and $\sum_{j>k \geq \ell}$ separately. If $j \leq \ell \leq k$, from (3.6) and Hölder's inequality, we deduce that for all 
$y \in \mathbb{R}^{n}$,

$$
\begin{aligned}
\mid\left(\chi_{k} * \varphi_{2^{-j}}\right. & \left.-\varphi_{2^{-j}}\right) * \psi_{2^{-j}} * f(y) \mid \\
& \lesssim 2^{2(j-k)} \int_{\mathbb{R}^{n}} \frac{2^{j n}}{\left(1+\left|2^{j} z\right|\right)^{L}}\left|\psi_{2^{-j}} * f(y-z)\right| d z \\
& \lesssim 2^{2(j-k)} \sum_{i=0}^{\infty} 2^{i(n-L)} f_{B\left(y, 2^{i-j}\right)}\left|\psi_{2^{-j}} * f(z)\right| d z \\
& \lesssim 2^{2(j-k)} \sum_{i=0}^{\infty} 2^{i(n-L)}\left\{f_{B\left(y, 2^{i-j}\right)}\left|\psi_{2^{-j}} * f(z)\right|^{q} d z\right\}^{1 / q} \\
& \lesssim 2^{2(j-k)} \sum_{i=0}^{\infty} 2^{i(n-L)} 2^{-j \alpha}\|f\|_{\dot{F}_{\infty, q}^{\alpha}\left(\mathbb{R}^{n}\right)} \\
& \lesssim 2^{2(j-k)} 2^{-j \alpha}\|f\|_{\dot{F}_{\infty, q}^{\alpha}\left(\mathbb{R}^{n}\right)},
\end{aligned}
$$

where we used the following trivial estimate that

$$
\left\{f_{B\left(y, 2^{i-j}\right)}\left|\psi_{2^{-j}} * f(z)\right|^{q} d z\right\}^{1 / q} \lesssim 2^{-j \alpha}\|f\|_{\dot{F}_{\infty, q}^{\alpha}\left(\mathbb{R}^{n}\right)} .
$$

Hence

$$
\begin{aligned}
& \left\{f_{B\left(x, 2^{-\ell}\right)} \sum_{k \geq \ell} 2^{k \alpha q}\left(\sum_{j \leq \ell}\left|\left(\varphi_{2^{-j}}-\chi_{k} * \varphi_{2^{-j}}\right) * \psi_{2^{-j}} * f(y)\right|\right)^{q} d y\right\}^{1 / q} \\
& \quad \lesssim\left\{f_{B\left(x, 2^{-\ell}\right)} \sum_{k \geq \ell} 2^{k \alpha q}\left(\sum_{j \leq \ell} 2^{2(j-k)} 2^{-j \alpha}\right)^{q} d y\right\}^{1 / q}\|f\|_{\dot{F}_{\infty, q}^{\alpha}\left(\mathbb{R}^{n}\right)} \\
& \lesssim\|f\|_{\dot{F}_{\infty, q}^{\alpha}\left(\mathbb{R}^{n}\right)} .
\end{aligned}
$$

If $\ell \leq j \leq k$, then, for all $y \in \mathbb{R}^{n}$,

$$
\begin{aligned}
\mid\left(\chi_{k} * \varphi_{2^{-j}}-\right. & \left.\varphi_{2^{-j}}\right) * \psi_{2^{-j}} * f(y) \mid \\
\lesssim & 2^{2(j-k)} M\left(\left|\psi_{2^{-j}} * f\right| \chi_{B\left(x, 2^{-\ell}\right)}\right)(y) \\
& \quad+\sum_{i \geq j-\ell} 2^{i(n-L)} f_{B\left(y, 2^{i-j}\right)}\left|\psi_{2^{-j}} * f(z)\right| d z \\
\lesssim & 2^{2(j-k)} M\left(\left|\psi_{2^{-j}} * f\right| \chi_{B\left(x, 2^{-\ell}\right)}\right)(y) \\
& +2^{2(j-k)} 2^{-j \alpha} 2^{(j-\ell)(n-L)}\|f\|_{\dot{F}_{\infty, q}^{\alpha}\left(\mathbb{R}^{n}\right)}
\end{aligned}
$$


and hence, by Hölder's inequality,

$$
\begin{aligned}
& \left\{f_{B\left(x, 2^{-\ell}\right)} \sum_{k \geq \ell} 2^{k \alpha q}\left[\sum_{\ell<j \leq k}\left|\left(\varphi_{2^{-j}}-\chi_{k} * \varphi_{2^{-j}}\right) * \psi_{2^{-j}} * f(y)\right|\right]^{q} d y\right\}^{1 / q} \\
& \quad \lesssim\left\{f_{B\left(x, 2^{-\ell}\right)} \sum_{k \geq \ell} 2^{k \alpha q}\left[\sum_{\ell<j \leq k} 2^{2(j-k)} M\left(\left|\psi_{2^{-j}} * f\right| \chi_{B\left(x, 2^{-\ell}\right)}\right)(y)\right]^{q} d y\right\}^{1 / q} \\
& \quad+\left\{f_{B\left(x, 2^{-\ell}\right)} \sum_{k \geq \ell} 2^{k \alpha q}\left[\sum_{\ell<j \leq k} 2^{2(j-k)} 2^{(n-L)(j-\ell)} 2^{-j \alpha}\right]^{q} d y\right\}^{1 / q}\|f\|_{\dot{F}_{\infty, q}^{\alpha}\left(\mathbb{R}^{n}\right)} \\
& \lesssim\left\{f_{B\left(x, 2^{-\ell}\right)} \sum_{k \geq \ell} 2^{k \alpha q}\left[\sum_{\ell<j \leq k} 2^{2(j-k)} \mid \psi_{2^{-j} * f(y) \mid} d y\right\}^{1 / q}+\|f\|_{\dot{F}_{\infty, q}^{\alpha}\left(\mathbb{R}^{n}\right)}\right. \\
& \lesssim\|f\|_{\dot{F}_{\infty, q}^{\alpha}\left(\mathbb{R}^{n}\right)} \cdot
\end{aligned}
$$

Similarly, if $j>k \geq \ell$, then we have

$$
\begin{aligned}
\mid\left(\chi_{k} * \varphi_{2^{-j}}-\right. & \left.\varphi_{2^{-j}}\right) * \psi_{2^{-j}} * f(y) \mid \\
\lesssim & M\left(\chi_{B\left(x, 2^{-\ell}\right)} M\left(\left|\psi_{2^{-j}} * f\right| \chi_{B\left(x, 2^{-\ell}\right)}\right)\right)(y) \\
& \quad+2^{-\alpha j} 2^{(j-\ell)(n-L)}\|f\|_{\dot{F}_{\infty, q}^{\alpha}\left(\mathbb{R}^{n}\right)},
\end{aligned}
$$

which further implies that

$$
\begin{array}{r}
\left\{f_{B\left(x, 2^{-\ell}\right)} \sum_{k \geq \ell} 2^{k \alpha q}\left(\sum_{j>k}\left|\left(\varphi_{2^{-j}}-\chi_{k} * \varphi_{2^{-j}}\right) * \psi_{2^{-j}} * f(y)\right|\right)^{q} d y\right\}^{1 / q} \\
\lesssim\|f\|_{\dot{F}_{\infty, q}^{\alpha}\left(\mathbb{R}^{n}\right)}
\end{array}
$$

This proves (3.4).

Now we consider the case $\alpha \in(2 N, 2 N+2)$ with $N \in \mathbb{N}$. Since the idea of the proof is similar to the case $\alpha \in(0,2)$, we only sketch the main steps. First we observe that $\dot{F}_{p, q}^{\alpha}\left(\mathbb{R}^{n}\right) \subset \dot{W}_{\text {loc }}^{2 N, 1}\left(\mathbb{R}^{n}\right)$, which follows from the lifting properties of Triebel-Lizorkin spaces (see $[\mathbf{1 3}]$ ) and the fact that $\dot{F}_{p, q}^{\alpha-2 N}\left(\mathbb{R}^{n}\right) \subset L_{\text {loc }}^{1}\left(\mathbb{R}^{n}\right)$ mentioned above. Moreover, similar to the above, $f \in \dot{F}_{p, q}^{\alpha}\left(\mathbb{R}^{n}\right)$ implies that the degrees of the polynomials $\left\{P_{i}\right\}_{i \in \mathbb{Z}}$ in (2.1) do not exceed $\lfloor\alpha-n / p\rfloor \leq 2 N+1$, and also that the 
polynomial $P_{f}$ is unique modulo a polynomial with degree no more than $\lfloor\alpha-n / p\rfloor \leq 2 N+1$; see [4, pp. 153-155] and [3]. In what follows, we set $\widetilde{f}:=f+P_{f}$ and let $\widetilde{R}_{N}\left(y ; x, 2^{-k}\right)$ be defined as in (1.2) with $f$ replaced by $\tilde{f}$.

Notice that for $i \in \mathbb{N}, f_{B(0,1)}|y|^{2 i} d y=\frac{n}{n+2 i}$. Then from (3.1), it follows that for all $x \in \mathbb{R}^{n}$,

$$
\begin{aligned}
f_{B\left(x, 2^{-k}\right)} \widetilde{R}_{N}\left(y ; x, 2^{-k}\right) d y & =\chi_{k} * \widetilde{f}(x)-\sum_{i=0}^{N} 2^{-2 i k} \frac{1}{L_{i}} \frac{n}{n+2 i} \Delta^{i} \widetilde{f}(x) \\
& =\sum_{j \in \mathbb{Z}}\left[\chi_{k} * \varphi_{2^{-j}}-\sum_{i=0}^{N} 2^{-2 i k} \frac{1}{L_{i}} \frac{n}{n+2 i} \Delta^{i} \varphi_{2^{-j}}\right] \\
& * \psi_{2^{-j}} * f(x) .
\end{aligned}
$$

We now consider $\sum_{j \leq k}$ and $\sum_{j>k}$ separately.

By an argument similar to (3.5), we see that, for any smooth function $\Phi$ on $\mathbb{R}$ and $N \in \mathbb{N}$,

(3.7) $\Phi(1)=\Phi(0)+\sum_{i=1}^{2 N+1} \frac{\Phi^{(i)}(0)}{i !}+\frac{1}{(2 N+1) !} \int_{0}^{1}(1-s)^{2 N+1} \Phi^{(2 N+2)}(s) d s$.

As above, choosing $\Phi(s):=\varphi\left(2^{j} x+s z\right)$ for $s \in[0,1]$ and $x, z \in \mathbb{R}^{n}$, we have

$$
\begin{aligned}
\varphi\left(2^{j} x+z\right)= & \varphi\left(2^{j} x\right)+(\nabla \varphi)\left(2^{j} x\right) z^{t}+\frac{1}{2 !} z\left(\nabla^{2} \varphi\right)\left(2^{j} x\right) z^{t}+\cdots \\
& +\frac{1}{(2 N+1) !} \int_{0}^{1}(1-s)^{2 N+1} z^{N+1}\left(\nabla^{2 N+2} \varphi\right)\left(2^{j} x+s z\right)\left(z^{t}\right)^{N+1} d s .
\end{aligned}
$$

Notice that in this expansion, except the terms $\frac{1}{L_{i}} \Delta^{i} \varphi\left(2^{j} x\right)\left|2^{j-k} z\right|^{2 i}$ for $0 \leq i \leq N$ and the last term, the other terms are harmonic and then have average 0 on any ball centered at 0 . When $j \leq k$, applying these 
facts, we conclude that, for all $x \in \mathbb{R}^{n}$,

$$
\begin{array}{rl}
\mid \chi_{k} & * \varphi_{2^{-j}}(x)-\sum_{i=0}^{N} 2^{-2 i k} \frac{1}{L_{i}} \frac{n}{n+2 i} \Delta^{i} \varphi_{2^{-j}}(x) \mid \\
& =\left|f_{B(0,1)} 2^{j n}\left[\varphi\left(2^{j} x+2^{j-k} z\right)-\sum_{i=0}^{N} \frac{1}{L_{i}} \Delta^{i} \varphi\left(2^{j} x\right)\left|2^{j-k} z\right|^{2 i}\right] d z\right| \\
& =\left|f_{B\left(0,2^{j-k}\right)} 2^{j n} \int_{0}^{1}(1-s)^{2 N+1} z^{N+1}\left(\nabla^{2 N+2} \varphi\right)\left(2^{j} x+s z\right)\left(z^{t}\right)^{N+1} d s d z\right| \\
& \lesssim 2^{2(N+1)(j-k)} \frac{2^{j n}}{\left(1+\left|2^{j} x\right|\right)^{L}},
\end{array}
$$

where $L \in \mathbb{N}$ is larger than $n$. Here the decay factor $2^{2(N+1)(j-k)}$ is crucial. Indeed, when $j \leq k$, we see that

$$
\begin{array}{r}
\left|\left(\chi_{k} * \varphi_{2^{-j}}-\sum_{i=0}^{N} 2^{-2 i k} \frac{1}{L_{i}} \frac{n}{n+2 i} \Delta^{i} \varphi_{2^{-j}}\right) * \psi_{2^{-j}} * f(x)\right| \\
\lesssim 2^{(2 N+2)(j-k)} M\left(\left|\psi_{2^{-j}} * f\right|\right)(x),
\end{array}
$$

while, when $j>k$, we also see that

$$
\begin{aligned}
\mid\left(\chi_{k} * \varphi_{2^{-j}}-\sum_{i=0}^{N} 2^{-2 i k} \frac{1}{L_{i}} \frac{n}{n+2 i}\right. & \left.\Delta^{i} \varphi_{2^{-j}}\right) * \psi_{2^{-j}} * f(x) \mid \\
& \lesssim 2^{2 N(j-k)} M \circ M\left(\left|\psi_{2^{-j}} * f\right|\right)(x) .
\end{aligned}
$$

Since $2 N<\alpha<2(N+1)$, for all $p \in(1, \infty)$ and $q \in(1, \infty]$, by exactly the same procedure as above, we conclude that $\left\|S_{\alpha, q}(\widetilde{f})\right\|_{L^{p}\left(\mathbb{R}^{n}\right)}$

$$
\begin{aligned}
& \lesssim\left\{\int_{\mathbb{R}^{n}}\left(\sum_{k \in \mathbb{Z}} 2^{k \alpha q}\left[\sum_{j \leq k} 2^{2(N+1)(j-k)} M\left(\left|\psi_{2^{-j}} * f\right|\right)(x)\right]^{q}\right)^{p / q} d x\right\}^{1 / p} \\
& +\left\{\int_{\mathbb{R}^{n}}\left(\sum_{k \in \mathbb{Z}} 2^{k \alpha q}\left[\sum_{j>k} 2^{2 N(j-k)} M \circ M\left(\left|\psi_{2^{-j}} * f\right|\right)(x)\right]^{q}\right)^{p / q} d x\right\}^{1 / p} \\
& \lesssim\|f\|_{\dot{F}_{p, q}^{\alpha}\left(\mathbb{R}^{n}\right)}
\end{aligned}
$$


When $2 N<\alpha<2(N+1)$, for all $p=\infty$ and $q \in(1, \infty]$, from a much more complicated argument, similar to the case $0<\alpha<2, p=\infty$ and $q \in(1, \infty]$, we also deduce that $\|\widetilde{f}\|_{S \dot{F}_{p, q}^{\alpha}\left(\mathbb{R}^{n}\right)} \lesssim\|f\|_{\dot{F}_{p, q}^{\alpha}\left(\mathbb{R}^{n}\right)}$. We omit the details. This finishes the proof of Theorem 3.1.

Theorem 3.2. Let $\alpha \in(0, \infty) \backslash 2 \mathbb{N}$ and $p, q \in(1, \infty]$. If $f \in S \dot{F}_{p, q}^{\alpha}\left(\mathbb{R}^{n}\right)$, then $f \in \dot{F}_{p, q}^{\alpha}\left(\mathbb{R}^{n}\right)$ and there exists a positive constant $C$, independent of $f$, such that

$$
\|f\|_{\dot{F}_{p, q}^{\alpha}\left(\mathbb{R}^{n}\right)} \leq C\|f\|_{S \dot{F}_{p, q}^{\alpha}\left(\mathbb{R}^{n}\right)} .
$$

The proof of Theorem 3.2 depends heavily on the following Calderón reproducing formulae.

Lemma 3.2. Let $\chi:=\frac{\chi_{B(0,1)}}{|B(0,1)|}, L \in \mathbb{Z}_{+} \cup\{-1\}$ and $N \in \mathbb{N}$.

(i) There exist $\phi, \psi \in \mathcal{S}\left(\mathbb{R}^{n}\right)$ satisfying that $\operatorname{supp} \phi \subset B(0,1), \int_{\mathbb{R}^{n}} \phi(x) x^{\gamma} d x=$ 0 for all $|\gamma| \leq L$ and $\operatorname{supp} \widehat{\psi} \subset\left\{\xi \in \mathbb{R}^{n}: 1 / 64 \leq|\xi| \leq 1 / 16\right\}$ such that, for all $\xi \in \mathbb{R}^{n} \backslash\{0\}$,

$$
\sum_{j \in \mathbb{Z}} \widehat{\psi}_{2^{-j}}(\xi) \widehat{\phi}_{2^{-j}}(\xi)\left[\widehat{\chi}_{2^{-j}}(\xi)-\widehat{\chi}_{2^{1-j}}(\xi)\right]=1 .
$$

Moreover, for every $f \in L_{\text {loc }}^{1}\left(\mathbb{R}^{n}\right) \cap \mathcal{S}^{\prime}\left(\mathbb{R}^{n}\right)$, there exist polynomials $\left\{P_{j}\right\}_{j \in \mathbb{Z}}$ and $P_{f}$ such that

$$
f+P_{f}=\lim _{i \rightarrow-\infty}\left\{\sum_{j=i}^{\infty} \phi_{2^{-j}} * \psi_{2^{-j}} *\left(f_{B\left(\cdot, 2^{-j}\right)}-f_{B\left(\cdot, 2^{1-j}\right)}\right)+P_{i}\right\}
$$

in $\mathcal{S}^{\prime}\left(\mathbb{R}^{n}\right)$.

(ii) There exist $\phi, \psi \in \mathcal{S}\left(\mathbb{R}^{n}\right)$ satisfying the same conditions as in (i) such that, for all $\xi \in \mathbb{R}^{n} \backslash\{0\}$,

$$
\begin{aligned}
\sum_{j \in \mathbb{Z}} \widehat{\psi}_{2^{-j}}(\xi) \widehat{\phi}_{2^{-j}}(\xi)\{ & {\left[\widehat{\chi}_{2^{-j}}(\xi)-\sum_{i=1}^{N} 2^{-2 i j} \frac{1}{L_{i}} \frac{n}{n+2 i}|\xi|^{2 i}\right] } \\
& \left.-\left[\widehat{\chi}_{2^{1-j}}(\xi)-\sum_{i=1}^{N} 2^{-2 i(j-1)} \frac{1}{L_{i}} \frac{n}{n+2 i}|\xi|^{2 i}\right]\right\}=1
\end{aligned}
$$


Moreover, for every $f \in \dot{W}_{\text {loc }}^{2 N, 1}\left(\mathbb{R}^{n}\right) \cap \mathcal{S}^{\prime}\left(\mathbb{R}^{n}\right)$, there exist polynomials $\left\{P_{j}\right\}_{j \in \mathbb{Z}}$ and $P_{f}$ such that

(3.11) $f+P_{f}=\lim _{m \rightarrow-\infty}\left\{\sum_{j=m}^{\infty} \phi_{2^{-j} * \psi_{2^{-j}} *}\left[\left(f_{B\left(\cdot, 2^{-j}\right)}-\sum_{i=1}^{N} 2^{-2 i j} \frac{1}{L_{i}} \frac{n}{n+2 i} \Delta^{i} f\right)\right.\right.$

$$
\left.\left.-\left(f_{B\left(\cdot, 2^{1-j}\right)}-\sum_{i=1}^{N} 2^{-2 i(j-1)} \frac{1}{L_{i}} \frac{n}{n+2 i} \Delta^{i} f\right)\right]+P_{m}\right\}
$$

in $\mathcal{S}^{\prime}\left(\mathbb{R}^{n}\right)$.

Proof: (i) It suffices to show (3.8). The proof of (3.9) follows from (3.8) and an argument similar to the arguments in [11, pp. 52-54].

First we show that there exists a positive constant $C_{0}$ such that for all $1 / 64 \leq|\xi| \leq 1 / 16$,

$$
\left|\widehat{\chi}(\xi)-\widehat{\chi}_{2}(\xi)\right| \geq C_{0}>0 \text {. }
$$

By $\left[7\right.$, p. 429], we know that $\widehat{\chi_{B(0,1)}}(\xi)=J_{n / 2}(2 \pi \xi) /|\xi|^{n / 2}$, where $J_{n / 2}$ is the Bessel function of order $n / 2$. Thus,

$$
\widehat{\chi}(\xi)=\frac{1}{|B(0,1)|} \frac{J_{n / 2}(2 \pi \xi)}{|\xi|^{n / 2}} \quad \text { and } \quad \widehat{\chi}_{2}(\xi)=\frac{1}{|B(0,1)|} \frac{J_{n / 2}(4 \pi \xi)}{|2 \xi|^{n / 2}} .
$$

Therefore,

$$
\begin{aligned}
\widehat{\chi}(\xi)-\widehat{\chi}_{2}(\xi)= & \frac{\pi^{n / 2}}{|B(0,1)| \Gamma(n / 2+1 / 2) \Gamma(1 / 2)} \\
& \times\left\{\int_{-1}^{1}\left[e^{2 \pi i|\xi| s}-e^{4 \pi i|\xi| s}\right]\left(1-s^{2}\right)^{n / 2-1 / 2} d s\right\} .
\end{aligned}
$$

Notice that if $1 / 64 \leq|\xi| \leq 1 / 16$ and $s \in[-1,1]$, then $4 \pi|\xi| s \in[-\pi / 4, \pi / 4]$ and hence $\cos (2 \pi|\xi| s) \geq \cos (4 \pi|\xi| s)$. Then we conclude that

$$
\begin{aligned}
\left|\widehat{\chi}(\xi)-\widehat{\chi}_{2}(\xi)\right| \geq & \frac{\pi^{n / 2}}{|B(0,1)| \Gamma(n / 2+1 / 2) \Gamma(1 / 2)} \\
& \times\left\{\int_{-1}^{1}[\cos (2 \pi|\xi| s)-\cos (4 \pi|\xi| s)]\left(1-s^{2}\right)^{n / 2-1 / 2} d s\right\} .
\end{aligned}
$$


By the fact that $1 / 64 \leq|\xi| \leq 1 / 16$ and $s \in[-1,1]$ again, we see that $\pi^{2}|\xi|^{2} s^{2} \geq 10 \pi^{4}|\xi|^{4} s^{4}$. Thus, by the Taylor expansion of the cosine function, we know that

$$
\cos (2 \pi|\xi| s)-\cos (4 \pi|\xi| s) \geq 5 \pi^{2}|\xi|^{2} s^{2}
$$

and hence

$$
\left|\widehat{\chi}(\xi)-\widehat{\chi}_{2}(\xi)\right| \geq \frac{5 \pi^{2}|\xi|^{2} \pi^{n / 2}}{|B(0,1)| \Gamma(n / 2+1 / 2) \Gamma(1 / 2)}\left\{\int_{-1}^{1} s^{2}\left(1-s^{2}\right)^{n / 2-1 / 2} d s\right\} .
$$

From the properties of Gamma functions (see [7, Appendix A]), it follows that

$$
\begin{gathered}
\frac{\pi^{n / 2}}{|B(0,1)| \Gamma(n / 2+1 / 2) \Gamma(1 / 2)}\left\{\int_{-1}^{1}\left(1-s^{2}\right)^{n / 2-1 / 2} d s\right\} \\
=\frac{n \Gamma(n / 2)}{2 \Gamma(n / 2+1 / 2) \Gamma(1 / 2)}\left\{\int_{0}^{1}(1-t)^{n / 2-1 / 2} t^{-1 / 2} d t\right\} \\
=\frac{n \Gamma(n / 2)}{2 \Gamma(n / 2+1 / 2) \Gamma(1 / 2)} \frac{\Gamma(n / 2+1 / 2) \Gamma(1 / 2)}{\Gamma(n / 2+1)}=1 .
\end{gathered}
$$

Thus,

$$
\begin{aligned}
\left|\widehat{\chi}(\xi)-\widehat{\chi}_{2}(\xi)\right| & \geq 5 \pi^{2}|\xi|^{2} \frac{\int_{-1}^{1} s^{2}\left(1-s^{2}\right)^{n / 2-1 / 2} d s}{\int_{-1}^{1}\left(1-s^{2}\right)^{n / 2-1 / 2} d s} \\
& =5 \pi^{2}|\xi|^{2} \frac{\int_{0}^{1} t^{1 / 2}(1-t)^{n / 2-1 / 2} d t}{\int_{0}^{1} t^{-1 / 2}(1-t)^{n / 2-1 / 2} d t} \\
& =5 \pi^{2}|\xi|^{2} \frac{\Gamma(3 / 2) \Gamma(n / 2+1 / 2) \Gamma(n / 2+1)}{\Gamma(1 / 2) \Gamma(n / 2+1 / 2) \Gamma(n / 2+2)} \\
& =\frac{5 \pi^{2}|\xi|^{2}}{n+2} .
\end{aligned}
$$

Therefore, for all $1 / 64 \leq|\xi| \leq 1 / 16$, we have

$$
\left|\widehat{\chi}(\xi)-\widehat{\chi}_{2}(\xi)\right| \geq 2^{-12} \frac{5 \pi^{2}}{n+2}>0,
$$

namely, (3.12) holds. 
For any fixed $L \in \mathbb{Z}_{+} \cup\{-1\}$, select a smooth function $\phi$ on $\mathbb{R}^{n}$ such that $\operatorname{supp} \phi \subset B(0,1), \int_{\mathbb{R}^{n}} \phi(x) x^{\gamma} d x=0$ for all $|\gamma| \leq L$, and $|\widehat{\phi}(\xi)| \geq C>0$ for all $1 / 64 \leq|\xi| \leq 1 / 16$, where $C$ is a positive constant. Then $\phi *\left(\chi-\chi_{2}\right) \in C_{c}^{\infty}\left(\mathbb{R}^{n}\right)$, has vanishing moments till order $L$ and satisfies that

$$
\left|\widehat{\phi}(\xi)\left[\widehat{\chi}(\xi)-\widehat{\chi}_{2}(\xi)\right]\right| \geq C>0
$$

for all $1 / 64 \leq|\xi| \leq 1 / 16$.

Let $g \in \mathcal{S}\left(\mathbb{R}^{n}\right)$ such that $\widehat{g}$ is nonnegative, $\operatorname{supp} \widehat{g} \subset\left\{\xi \in \mathbb{R}^{n}: 1 / 64 \leq\right.$ $|\xi| \leq 1 / 16\}$ and $\widehat{g}(\xi) \geq C>0$ if $3 / 128 \leq|\xi| \leq 7 / 128$, where $C$ is a positive constant. Let

$$
F:=\sum_{j \in \mathbb{Z}} \widehat{g}\left(2^{-j} \cdot\right)
$$

Then $F$ is a bounded smooth function satisfying that $F(\xi) \geq C>0$ for all $\xi \neq 0$ and $F\left(2^{j} \cdot\right) \equiv F$.

Now define $h:=\widehat{g} / F$. Then $h \in \mathcal{S}\left(\mathbb{R}^{n}\right), \operatorname{supp} h \subset\left\{\xi \in \mathbb{R}^{n}:\right.$ $1 / 64 \leq|\xi| \leq 1 / 16\}, h(\xi) \geq C>0$ for all $3 / 128 \leq|\xi| \leq 7 / 128$, and $\sum_{j \in \mathbb{Z}} h\left(2^{-j} \xi\right)=1$ for all $\xi \neq 0$. By (3.14), we define a Schwartz function $\psi$ by setting $\widehat{\psi}:=h\left\{\widehat{\phi}\left[\widehat{\chi}-\widehat{\chi}_{2}\right]\right\}^{-1}$. Then

$$
\operatorname{supp} \widehat{\psi} \subset\left\{\xi \in \mathbb{R}^{n}: 1 / 64 \leq|\xi| \leq 1 / 16\right\}
$$

and, for all $\xi \in \mathbb{R}^{n} \backslash\{0\}$,

$$
\sum_{j \in \mathbb{Z}} \widehat{\psi}_{2^{-j}}(\xi) \widehat{\phi}_{2^{-j}}(\xi)\left[\widehat{\chi}_{2^{-j}}(\xi)-\widehat{\chi}_{2^{1-j}}(\xi)\right]=\sum_{j \in \mathbb{Z}} \widehat{h}\left(2^{-j} \xi\right)=1,
$$

which completes the proof of (i).

(ii) Similar to the argument in (i), it suffices to show that there exists a positive constant $C_{0}$ such that, for all $1 / 64 \leq|\xi| \leq 1 / 16$,

$$
\left|\left[\widehat{\chi}(\xi)-\sum_{i=1}^{N} \frac{1}{L_{i}} \frac{n}{n+2 i}|\xi|^{2 i}\right]-\left[\widehat{\chi}_{2}(\xi)-\sum_{i=1}^{N} 2^{2 i} \frac{1}{L_{i}} \frac{n}{n+2 i}|\xi|^{2 i}\right]\right| \geq C_{0}>0 .
$$

From (3.13), we deduce that, for all $1 / 64 \leq|\xi| \leq 1 / 16$,

$$
\left|\widehat{\chi}(\xi)-\widehat{\chi}_{2}(\xi)\right| \geq \frac{5 \pi^{2}|\xi|^{2}}{n+2},
$$


while

$$
\begin{aligned}
& \left.\left|\sum_{i=1}^{N} 2^{2 i} \frac{1}{L_{i}} \frac{n}{n+2 i}\right| \xi\right|^{2 i}-\sum_{i=1}^{N} \frac{1}{L_{i}} \frac{n}{n+2 i}|\xi|^{2 i} \mid \\
& \quad \leq|\xi|^{2} \sum_{i=1}^{N} \frac{\left(2^{2 i}-1\right) n}{n+2 i} \frac{2^{-4(2 i-2)}}{\sum_{m_{1}+\cdots+m_{n}=i}\left(2 m_{1}\right) ! \cdots\left(2 m_{n}\right) !} \leq 4|\xi|^{2} /(n+2) .
\end{aligned}
$$

Thus, (3.15) holds in this case, which completes the proof of (ii) and hence Lemma 3.2 .

Proof of Theorem 3.2: We first consider the case $\alpha \in(0,2)$. Let $f \in$ $S \dot{F}_{p, q}^{\alpha}\left(\mathbb{R}^{n}\right)$. By Lemma 3.2(i) and $f \in L_{\text {loc }}^{1}\left(\mathbb{R}^{n}\right) \cap \mathcal{S}^{\prime}\left(\mathbb{R}^{n}\right)$, we conclude that

$$
\begin{aligned}
f & =\sum_{k \in \mathbb{Z}} \phi_{2^{-k}} * \psi_{2^{-k}} *\left(\chi_{2^{-k}}-\chi_{2^{-k+1}}\right) * f \\
& =\sum_{k \in \mathbb{Z}} \phi_{2^{-k}} * \psi_{2^{-k}} *\left(f_{B\left(\cdot, 2^{-k}\right)}-f_{B\left(\cdot, 2^{1-k}\right)}\right),
\end{aligned}
$$

which, modulo polynomials, holds in $\mathcal{S}^{\prime}\left(\mathbb{R}^{n}\right)$. Here $\phi$ and $\psi$ are as in Lemma 3.2(i). Let $\varphi$ be as in (2.1). For $k \in \mathbb{Z}$, we have

$$
\varphi_{2^{-k}} * f=\sum_{j \in \mathbb{Z}} \varphi_{2^{-k}} * \phi_{2^{-j}} * \psi_{2^{-j}} *\left(f_{B\left(\cdot, 2^{-j}\right)}-f_{B\left(\cdot, 2^{1-j}\right)}\right) .
$$

Notice that for all $k, j \in \mathbb{Z}$, and $x \in \mathbb{R}^{n}$,

$$
\begin{aligned}
\left|\varphi_{2^{-k}} * \phi_{2^{-j}} * \psi_{2^{-j}}(x)\right| & =\left|\varphi_{2^{-k}} *(\phi * \psi)_{2^{-j}}(x)\right| \\
& \lesssim 2^{-s|j-k|} \frac{2^{n(\min (j, k))}}{\left(1+\left|2^{\min (j, k)} x\right|\right)^{L}}
\end{aligned}
$$

where $s, L$ can be chosen large enough as we need; see, for example, [18, Lemma 2.2]. Thus,

$$
\left|\varphi_{2^{-k}} * \phi_{2^{-j}} * \psi_{2^{-j}} * g\right|=\left|\varphi_{2^{-k}} *(\phi * \psi)_{2^{-j}} * g\right| \lesssim 2^{-2|j-k|} M(g) .
$$


Therefore, when $p \in(1, \infty)$, from Definition 2.1, Hölder's inequality and the Fefferman-Stein vector-valued maximal inequality, we infer that

$$
\begin{aligned}
\|f\|_{\dot{F}_{p, q}^{\alpha}\left(\mathbb{R}^{n}\right)} & \lesssim\left\|\left\{\sum_{k \in \mathbb{Z}} 2^{k \alpha q}\left|\sum_{j \leq k} 2^{-2(k-j)} M\left(f_{B\left(\cdot, 2^{-j}\right)}-f_{B\left(\cdot, 2^{1-j}\right)}\right)\right|^{q}\right\}^{1 / q}\right\|_{L^{p}\left(\mathbb{R}^{n}\right)} \\
& +\left\|\left\{\sum_{k \in \mathbb{Z}} 2^{k \alpha q}\left|\sum_{j>k} 2^{-2(j-k)} M\left(f_{B\left(\cdot, 2^{-j}\right)}-f_{B\left(\cdot, 2^{1-j}\right)}\right)\right|^{q}\right\}^{1 / q}\right\|_{L^{p}\left(\mathbb{R}^{n}\right)} \\
& \lesssim\left\|\left\{\sum_{j \in \mathbb{Z}} 2^{j \alpha q}\left[M\left(f_{B\left(\cdot, 2^{-j}\right)}-f_{B\left(\cdot, 2^{1-j}\right)}\right)\right]^{q}\right\}^{1 / q}\right\| \|_{L^{p}\left(\mathbb{R}^{n}\right)} \\
& \lesssim\left\|\left\{\sum_{j \in \mathbb{Z}} 2^{j \alpha q}\left|f_{B\left(\cdot, 2^{-j}\right)}-f_{B\left(\cdot, 2^{1-j}\right)}\right|^{q}\right\}^{1 / q}\right\| \|_{L^{p}\left(\mathbb{R}^{n}\right)} \\
& \lesssim\left\|\left\{\sum_{j \in \mathbb{Z}} 2^{j \alpha q}\left|f_{B\left(\cdot, 2^{-j}\right)}-f\right|^{q}\right\}^{1 / q}\right\|\left\|_{L^{p}\left(\mathbb{R}^{n}\right)}\right\| S_{\alpha, q}(f) \|_{L^{p}\left(\mathbb{R}^{n}\right)}
\end{aligned}
$$

When $p=\infty$, we need to show that

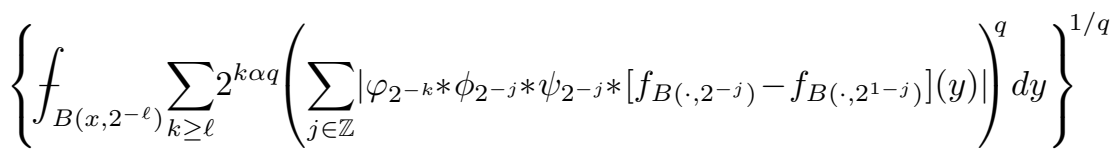

is controlled by $\|f\|_{S \dot{F}_{\infty, q}^{\alpha}\left(\mathbb{R}^{n}\right)}$ uniformly in $x \in \mathbb{R}^{n}$ and $\ell \in \mathbb{Z}$. The proof of this is quite similar to that of (3.4). Indeed, we consider $\sum_{j<\ell \leq k}$, $\sum_{\ell \leq j \leq k}$ and $\sum_{j>k \geq \ell}$ separately. With the help of (3.16) and some necessary calculus, we arrive at $\|f\|_{\dot{F}_{\infty, q}^{\alpha}\left(\mathbb{R}^{n}\right)} \lesssim\|f\|_{S \dot{F}_{\infty, q}^{\alpha}\left(\mathbb{R}^{n}\right)}$. We omit the details. 
When $\alpha \in(2 N, 2(N+1))$, by Lemma 3.2(ii), we see that

$$
\begin{aligned}
f=\sum_{k \in \mathbb{Z}} \phi_{2^{-k}} * \psi_{2^{-k}} * & {\left[f_{B\left(\cdot, 2^{-k}\right)}-\sum_{i=1}^{N} 2^{-2 i j} \frac{1}{L_{i}} \frac{n}{n+2 i} \Delta^{i} f\right.} \\
& \left.-f_{B\left(\cdot, 2^{1-k}\right)}+\sum_{i=1}^{N} 2^{-2 i(j-1)} \frac{1}{L_{i}} \frac{n}{n+2 i} \Delta^{i} f\right] .
\end{aligned}
$$

By (3.16) with $s=2(N+1)$ and $L>n$, repeating the above argument for the case $\alpha \in(0,2)$, we then conclude that $\|f\|_{\dot{F}_{p, q}^{\alpha}\left(\mathbb{R}^{n}\right)} \lesssim\|f\|_{S \dot{F}_{p, q}^{\alpha}\left(\mathbb{R}^{n}\right)}$. This finishes the proof of Theorem 3.2.

\section{Besov spaces and some remarks}

In this section, we first establish a similar characterization for Besov spaces and then make some remarks for the case $\alpha \in 2 \mathbb{N}$.

Let $N \in \mathbb{N} \cup\{0\}, \alpha \in(2 N, 2 N+2)$ and $p, q \in(0, \infty]$. The space $S \dot{B}_{p, q}^{\alpha}\left(\mathbb{R}^{n}\right)$ of Besov type is defined as the collection of functions $f \in$ $\dot{W}_{\text {loc }}^{2 N, 1}\left(\mathbb{R}^{n}\right) \cap \mathcal{S}^{\prime}\left(\mathbb{R}^{n}\right)$ such that

$\|f\|_{S \dot{B}_{p, q}^{\alpha}\left(\mathbb{R}^{n}\right)}:=\left\{\sum_{k \in \mathbb{Z}} 2^{k \alpha q}\left\|f_{B\left(\cdot, 2^{-k}\right)} R_{N}\left(y ; \cdot, 2^{-k}\right) d y\right\|_{L^{p}\left(\mathbb{R}^{n}\right)}^{q}\right\}^{1 / q}<\infty$.

Here $\dot{W}_{\text {loc }}^{0,1}\left(\mathbb{R}^{n}\right)=L_{\text {loc }}^{1}\left(\mathbb{R}^{n}\right), R_{N}$ with $N \geq 1$ is as in (1.2) and, for all $x, y \in \mathbb{R}^{n}$,

$$
R_{0}\left(y ; x, 2^{-k}\right):=f(y)-f(x) .
$$

Also the space $S B_{p, q}^{\alpha}\left(\mathbb{R}^{n}\right)$ is similarly defined as above.

Then Theorems 3.1 and 3.2 admit Besov space versions; indeed, by similar arguments, Theorems 3.1 and 3.2 still hold with spaces $\dot{F}_{p, q}^{\alpha}\left(\mathbb{R}^{n}\right)$ and $S \dot{F}_{p, q}^{\alpha}\left(\mathbb{R}^{n}\right)$ replaced by Besov spaces $\dot{B}_{p, q}^{\alpha}\left(\mathbb{R}^{n}\right)$ and $S \dot{B}_{p, q}^{\alpha}\left(\mathbb{R}^{n}\right)$ and, moreover, with the indices $\alpha, p$ and $q$ replaced, respectively, by $\alpha \in$ $(0, \infty) \backslash 2 \mathbb{N}, p \in(1, \infty]$ and $q \in(0, \infty]$. Namely, we have the following characterization on Besov spaces.

Theorem 4.1. Let $\alpha \in(0, \infty) \backslash 2 \mathbb{N}, p \in(1, \infty]$ and $q \in(0, \infty]$. Then $\dot{B}_{p, q}^{\alpha}\left(\mathbb{R}^{n}\right)=S \dot{B}_{p, q}^{\alpha}\left(\mathbb{R}^{n}\right)$, with equivalent norms, and $B_{p, q}^{\alpha}\left(\mathbb{R}^{n}\right)=S B_{p, q}^{\alpha}\left(\mathbb{R}^{n}\right)$, with equivalent norms.

It should be pointed out that $B_{p, q}^{\alpha}\left(\mathbb{R}^{n}\right) \subset S B_{p, q}^{\alpha}\left(\mathbb{R}^{n}\right)$ when $\alpha \in(0, \infty)$ and $p, q \in[1, \infty]$ was obtained by Wheeden $[\mathbf{1 5}$, Theorem 5$]$ via a totally different approach. 
Finally, we make some remarks. The first remark is on the missing indexes $\alpha \in 2 \mathbb{N}$ in Theorems 1.1 and 1.2 while the second one is on the higher order Besov and Triebel-Lizorkin spaces on metric measure spaces.

Remark 4.1. We point out that when $\alpha \in 2 \mathbb{N}$, it was proved in [1] that a variant of Theorems 1.1 and 1.2 when $p \in(1, \infty)$ and $q=2$ still holds. However, it is not clear that when $\alpha \in 2 \mathbb{N}$, whether there exists a similar variant of Theorems 1.1 and 1.2 when $q \neq 2$ and Theorem 4.1 for all $q \in(0, \infty]$. Indeed, as pointed out in [1], $S_{2,2}$-function as in (1.1) fails to characterize $\dot{F}_{p, 2}^{2}\left(\mathbb{R}^{n}\right)=\dot{W}^{2, p}\left(\mathbb{R}^{n}\right)$. To overcome this drawback, Alabern, Mateu and Verdera [1] then introduced a variant of (1.1) to characterize $\dot{F}_{p, 2}^{2}\left(\mathbb{R}^{n}\right)$. Precisely, for $N \in \mathbb{Z}_{+}, k \in \mathbb{Z}$, and $x, y \in \mathbb{R}^{n}$, let

$$
\begin{aligned}
\bar{R}_{N}\left(y ; x, 2^{-k}\right):=f(y) & -f(x)-\sum_{j=1}^{N-1} \frac{1}{L_{j}} \Delta^{j} f(x)|y-x|^{2 j} \\
& -\frac{1}{L_{N}}\left(f_{B\left(x, 2^{-k}\right)} \Delta^{N} f(z) d z\right)|y-x|^{2 N},
\end{aligned}
$$

where $L_{N}$ is as in (1.2). Let $\bar{S}_{\alpha, q}(f)$ be as in (1.1) with $R_{N}$ replaced by $\bar{R}_{N}$ and, similarly, the spaces $\bar{S} \dot{F}_{p, q}^{\alpha}\left(\mathbb{R}^{n}\right)$ for $\alpha \in[2 N, 2 N+2)$ and $p, q \in(1, \infty]$ are similarly defined to the spaces $S \dot{F}_{p, q}^{\alpha}\left(\mathbb{R}^{n}\right)$. Then it was proved in [1] that $\bar{S} \dot{F}_{p, 2}^{2 N}\left(\mathbb{R}^{n}\right)=\dot{F}_{p, 2}^{2 N}\left(\mathbb{R}^{n}\right)=\dot{W}^{2 N, p}\left(\mathbb{R}^{n}\right)$ for $N \in \mathbb{N}$ and $p \in(1, \infty)$.

When $\alpha \in(2 N, 2 N+2)$ and $p, q \in(1, \infty]$, by modifying the proofs in Section 3 , we can also show that $\bar{S} \dot{F}_{p, q}^{\alpha}\left(\mathbb{R}^{n}\right)=\dot{F}_{p, q}^{\alpha}\left(\mathbb{R}^{n}\right)$ with equivalent norms. But our above proof can only show $\bar{S} \dot{F}_{p, q}^{2 N}\left(\mathbb{R}^{n}\right) \subset \dot{F}_{p, q}^{2 N}\left(\mathbb{R}^{n}\right)$ for $N \in \mathbb{Z}_{+}$and $p, q \in(1, \infty]$. It is still unknown whether the relation $\dot{F}_{p, q}^{2 N}\left(\mathbb{R}^{n}\right) \subset \bar{S} \dot{F}_{p, q}^{2 N}\left(\mathbb{R}^{n}\right)$ is still true for $N \in \mathbb{Z}_{+}$and $p, q \in(1, \infty]$ but $q \neq 2$ or not.

Remark 4.2. On a metric measure space $\mathcal{X}$ satisfying a doubling property, when $\alpha \in(0,1)$ and $p, q \in(n /(n+\alpha), \infty]$, Triebel-Lizorkin spaces $\dot{F}_{p, q}^{\alpha}(\mathcal{X})$ are well defined and enjoy several important properties; see, for example, $[\mathbf{1 7}],[\mathbf{1 0}],[\mathbf{6}],[\mathbf{8}],[\mathbf{9}],[\mathbf{1 9}]$ and their references. But when $\alpha \geq 1$, a reasonable definition for Triebel-Lizorkin spaces $\dot{F}_{p, q}^{\alpha}(\mathcal{X})$ is still not available. However, by exactly the same way as in Definition 1.1, we can define the spaces $S \dot{F}_{p, q}^{\alpha}(\mathcal{X})$ of Triebel-Lizorkin type for $\alpha \in(0,2)$ and $p, q \in(1, \infty]$. It would be interesting to know whether the following are true: 
a) when $\alpha \in(0,1)$ and $p, q \in(1, \infty], S \dot{F}_{p, q}^{\alpha}(\mathcal{X})=\dot{F}_{p, q}^{\alpha}(\mathcal{X})$ ?

b) when $\alpha \in[1,2)$ and $p, q \in(1, \infty]$, does $S \dot{F}_{p, q}^{\alpha}(\mathcal{X})$ satisfy some of the important properties of $\dot{F}_{p, q}^{\alpha}\left(\mathbb{R}^{n}\right)$ ?

Similar questions are also asked in regards to spaces $S \dot{B}_{p, q}^{\alpha}(\mathcal{X})$ of Besov type with $\alpha \in(0,2), p \in(1, \infty]$ and $q \in(0, \infty]$.

The answers of these questions may depend on the geometry of the underlying metric measure spaces $\mathcal{X}$.

Acknowledgements. The authors would like to thank Professor Joan Verdera and Professor Hans Triebel for some helpful discussions on the subject of this paper. They also sincerely wish to express their deeply thanks to the referee for her/his very carefully reading and also her/his several careful, valuable and suggestive remarks which improve the presentation of this article.

\section{References}

[1] R. Alabern, J. Mateu, and J. Verdera, A new characterization of Sobolev spaces on $\mathbb{R}^{n}$, Math. Ann. 354(2) (2012), 589-626. DOI : $10.1007 / \mathrm{s} 00208-011-0738-0$.

[2] C. Fefferman And E. M. Stein, Some maximal inequalities, Amer. J. Math. 93 (1971), 107-115.

[3] M. Frazier AND B. JAWERTh, Decomposition of Besov spaces, Indiana Univ. Math. J. 34(4) (1985), 777-799. DOI: 10.1512/iumj. 1985.34 .34041$.

[4] M. Frazier And B. Jawerth, A discrete transform and decompositions of distribution spaces, J. Funct. Anal. 93(1) (1990), 34-170. DOI: $10.1016 / 0022-1236$ (90) 90137-A.

[5] M. Frazier, B. Jawerth, AND G. Weiss, "Littlewood-Paley theory and the study of function spaces", CBMS Regional Conference Series in Mathematics 79, Published for the Conference Board of the Mathematical Sciences, Washington, DC; by the American Mathematical Society, Providence, RI, 1991.

[6] A. Gogatishvili, P. Koskela, and Y. Zhou, Characterizations of Besov and Triebel-Lizorkin spaces on metric measure spaces, Forum Math. DOI: 10.1515/form.2011.135.

[7] L. Grafakos, "Classical Fourier analysis", Second edition, Graduate Texts in Mathematics 249, Springer, New York, 2008. 
[8] P. Koskela, D. YAng, And Y. Zhou, A characterization of Hajłasz-Sobolev and Triebel-Lizorkin spaces via grand LittlewoodPaley functions, J. Funct. Anal. 258(8) (2010), 2637-2661. DOI: 10.1016/j.jfa.2009.11.004.

[9] P. Koskela, D. Yang, And Y. Zhou, Pointwise characterizations of Besov and Triebel-Lizorkin spaces and quasiconformal mappings, Adv. Math. 226(4) (2011), 3579-3621. DOI: 10.1016/j.aim.2010. 10.020 .

[10] D. MÜLler ANd D. YANG, A difference characterization of Besov and Triebel-Lizorkin spaces on RD-spaces, Forum Math. 21(2) (2009), 259-298. DOI : 10.1515/FORUM. 2009.013.

[11] J. PeEtre, "New thoughts on Besov spaces", Duke University Mathematics Series 1, Mathematics Department, Duke University, Durham, N.C., 1976.

[12] E. M. Stein, "Singular integrals and differentiability properties of functions", Princeton Mathematical Series 30, Princeton University Press, Princeton, N.J. 1970.

[13] H. Triebel, "Theory of function spaces", Monographs in Mathematics 78, Birkhäuser Verlag, Basel, 1983. DOI: 10.1007/978-30346-0416-1.

[14] H. Triebel, "Theory of function spaces. II", Monographs in Mathematics 84, Birkhäuser Verlag, Basel, 1992. DOI: 10.1007/978-30346-0419-2.

[15] R. L. Wheeden, Lebesgue and Lipschitz spaces and integrals of the Marcinkiewicz type, Studia Math. 32 (1969), 73-93.

[16] R. L. Wheeden, A note on a generalized hypersingular integral, Collection of articles honoring the completion by Antoni Zygmund of 50 years of scientific activity, I, Studia Math. 44 (1972), 17-26.

[17] D. YAng, New characterizations of Hajłasz-Sobolev spaces on metric spaces, Sci. China Ser. A 46(5) (2003), 675-689. DOI: 10.1360/ $02 y s 0343$.

[18] D. Yang And W. Yuan, A new class of function spaces connecting Triebel-Lizorkin spaces and $Q$ spaces, J. Funct. Anal. 255(10) (2008), 2760-2809. DOI: 10.1016/j.jfa.2008.09.005.

[19] D. Yang And Y. Zhou, New properties of Besov and Triebel-Lizorkin spaces on RD-spaces, Manuscripta Math. 134(1-2) (2011), 59-90. DOI: $10.1007 / \mathrm{s} 00229-010-0384-\mathrm{y}$. 
Dachun Yang and Wen Yuan:

School of Mathematical Sciences

Beijing Normal University

Laboratory of Mathematics and Complex Systems

Ministry of Education

Beijing 100875

People's Republic of China

E-mail address: dcyang@bnu.edu.cn

E-mail address: wenyuan@bnu.edu.cn

Yuan Zhou:

Department of Mathematics

Beijing University of Aeronautics and Astronautics

Beijing 100083

People's Republic of China

E-mail address: yuanzhou@buaa.edu.cn

and

Department of Mathematics and Statistics

P. O. Box 35 (MaD)

FI-40014, University of Jyväskylä

Finland

Primera versió rebuda el 26 d'agost de 2011, darrera versió rebuda el 29 de març de 2012. 